\title{
FAULT DETECTION AND DIAGNOSIS INGEARS USING WAVELET ENVELOPED POWER SPECTRUM AND ANN
}

\author{
M. Lokesha ${ }^{1}$, Manik Chandra Majumder ${ }^{2}$, K. P. Ramachandran ${ }^{3}$, Khalid Fathi Abdul Raheem ${ }^{4}$ \\ 1,3,4 Department of Mechanical Engineering, Caledonian College of Engineering, OMAN,lokjay251@yahoo.com, \\ ${ }^{2}$ Department of Mechanical Engineering, National Institute of Technology, Durgapur, INDIA,lokjay@gmail.com
}

\begin{abstract}
In this work, automatic detection and diagnosis of gear condition monitoring technique is presented. The vibration signals in time domain wereobtained from a fault simulator apparatus from a healthy gear and an induced faulty gear. These time domain signals were processed using Laplace and Morlet wavelet based enveloped power spectrum to detect the faults in gears. The vibration signals obtained were filtered to enhance the signal components before the application of wavelet analysis. The time and frequency domain features extracted from Laplace wavelet based wavelet transform are used as input to ANN for gear fault classification. Genetic algorithm was used to optimize the wavelet and ANN classification parameters. The result shows the successful classification of ANN test process.
\end{abstract}

Index Terms:Continuous wavelet transform, Envelope power spectrum, Wavelet, Filtering, ANN.

\section{INTRODUCTION}

Gears are commonly used component for the transmission of mechanical power and variation of speed in machines. The condition monitoring of the machine with a reliable system can identify the fault at an early stage in order to avoid any failure in the machines [1]. Gear failure is one of the most common failures in the rotating machines which lead to economic losses. Therefore it is very important to detect gear failure $[2,3]$.

The defects which are usually found in gear box includes the presence of crack in one or more of the gear tooth, chipping of teeth, geometrical imperfection etc. Vibration based condition monitoring techniques is commonly used for the fault detection and diagnosis in mechanical components like gears, bearings etc.[4,5].Vibration signals are analysed using different techniques such as time domain, frequency domain and time-frequency domain techniques [6-8]. The impact produced by the faulty gear may result in non-stationary signal, which contain abundant information about faults; therefore, it is important to analyze the non-stationary signals. The non-stationary nature of the signal suggests the use of time-frequency techniques, which make it possible to look at the time evolution of the signal's frequency content $[9,10]$.

FFT based condition monitoring techniques are not suitable for non-stationary signal, which assumes the analyzed signal to be strictly periodic [11].The non-stationary nature in the signal can be analyzed by the wavelet transform (WT), which provides powerful multi resolution analysis in both time and frequency domains. The time and frequency analysis of signals makes the WT as a favored tool to extract the transient features of non-stationary vibrations signals compared to fast Fourier transform spectrum [12-14].Wavelet coefficients, which are obtained by the wavelet analysis indicate the correlation of the signal with the particular wavelet. In order to extract the fault features of the signal appropriate wavelet base function should be selected.

Laplace wavelet is a complex, single sided damped exponential formulated as an impulse response of a single mode system to be similar to data features commonly encountered in health monitoring tasks. It has been applied to the vibration analysis of an aircraft for aerodynamic and structural testing [15] and to diagnose the wear of the intake valve of an internal combustion engine [16]. Kurtosis is defined as the fourth central cumulant divided by the square of the variance of the probability distribution [17]. A high kurtosis values indicates high impulsive content of the signal with more sharpness in the signal intensity distribution, based on maximum kurtosis concept, the fault detection is enhanced, by optimizing the wavelet features [18-20].

A neural network is a massively parallel distributed processor that has a natural tendency for storing knowledge and making it available for use. Artificial neural network (ANN) is a type of artificial intelligence, which has nonlinear information processing devices, built from interconnected elementary processing devices called neurons. Among all kinds of intelligent diagnosis methods, pattern recognition based on an Artificial Neural Network (ANN) has been widely used because of its power in self- organizing, unsupervisedlearning, and nonlinear pattern classification [21].The artificial 
neuron receives signals analogous to the natural electrochemical signals. The way information is processed and intelligence is stored depends on the architecture and algorithms of ANN.

Expert system and artificial intelligence are playing significant role in automated fault diagnosis and machine learning. Machine learning using the concepts of Artificial Neural Network (ANN) have become very popular and known for exploiting non-linear pattern classification properties, offering automatic detection and identification of gearbox fault conditions. It offers an advantage that they do not require an in-depth knowledge of the behavior of the system [22].

Artificial neural network (ANN), support vector machine (SVM) and Fuzzy classifier are widely used as classification tool [23].The most popular feed forward, multi-layer perceptronneural network, is frequently exploited in fault diagnosis systems and it has found an immense popularity in condition monitoring applications[24].

In this paper, Wiener filter is used to enhance the fault signal components in the vibration signal and wavelet envelope power spectrum is used to detect localized gear tooth defects. Vibration signals were collected from the experimental setups with induced faults. The second part includes the application of wavelet analysis as a feature extraction method combined with the neural network classifier for automatic detection and diagnosis of the gear fault.

This paper is organized as follows: section 2 covers theory on the wiener filter and wavelet transform, section 3 explains the experimental setups and procedure, section 4 discusses the implementation of the enveloped wavelet power spectrum forgear fault detection, section5 covers feature extraction method combined with ANN as classifier and the conclusions are presented in section 6 .

\section{WAVELET POWER SPECTRUM}

\subsection{Filtering}

The filtering theory works on the concept of minimizing the difference between the filtered output and the desired output. The Filter uses the least mean square approach to minimize, which adjusts the filter coefficients to reduce the square of the difference between the desired and actual waveform after filtering.

The concept of Wiener filter approach is represented by schematic diagram as depicted in Figure 1.

Input $\mathrm{A}(\mathrm{n}) \quad$ output $\mathrm{B}(\mathrm{n}) \quad$ error $\mathrm{E}(\mathrm{n})$

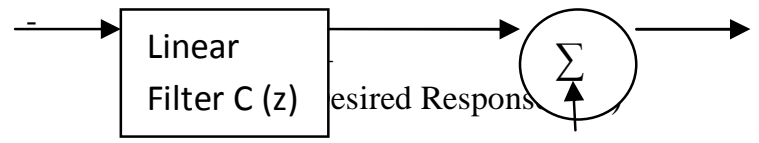

Figure 1
The linear process $\mathrm{C}(\mathrm{z})$ uses the concept of finite impulse response (FIR) filters to filter the input waveform A(n)having both signal and noise.

The FIR filters are implemented using convolution equation

$$
B(n)=\sum_{k=1}^{M} i(k) A(n-k)
$$

Where, the impulse response of the linear filter is represented by $i(k)$. The output of the filter, B $(n)$, can be thought of as an estimate of the desired signal, $D(n)$. The difference between the estimate and desired signal'E $(n)^{\prime}$ is determined by subtraction

$$
E(n)=D(n)-\mathrm{B}(n)
$$

To minimize error signal' $\mathrm{E}(n)^{\prime}$ the least mean square algorithm is used.

$\mathrm{E}(n)$ can be written as:

$$
\mathrm{E}(n)=\mathrm{D}(n)-\mathrm{B}(n)=\mathrm{D}(n)-\sum_{k=1}^{M} i(k) A(n-k)(2)
$$

Where $M$ is the length of the FIR filter. In fact, it is the sum of $E(n)^{2}$ which is to be minimized. Squaring of the elements ' $E$ $(n)^{2}$ 'leads to a quadratic function of the FIR filter coefficients, $i(\mathrm{k})$, which contains the autocorrelation and cross correlation elements.

In order to Minimize the error function with respect to the FIR filtercoefficients, take derivatives with respect to $i(k)$ and set them to zero. This represents a series of Mequations that must be solved simultaneouslyto solve for the FIR coefficients. The matrix expression for these simultaneous equations is known as the Wiener-Hopfequation and is abasic component of Wiener filter theory [25].

\subsection{Enveloped wavelet power spectrum}

Wavelet analysis can use different types of probing functions, but the family always consists of enlarged or compressed versions of the basic function, as well as translations. Mathematically, the wavelet transform of a finite energy signal $\mathrm{x}(\mathrm{t})$ with the analyzing wavelet $\psi$, leads to the definition of continuous wavelet transform as given in equation (3).

$$
\mathrm{W}(\mathrm{a}, \mathrm{b})=\int_{-\infty}^{\infty} x(t) \frac{1}{\sqrt{|a|}} \psi *\left(\frac{t-b}{a}\right) d t
$$

Where, $b$ (dilation parameter) acts to translate the function across $\mathrm{x}(\mathrm{t})$ and the variable $a$ (scaling parameter) acts to vary the time scale of the wavelet function $\psi$. The $*$ indicates the operation of complex conjugate and the normalizing parameter 
$1 / \sqrt{a}$ ensures that the energy is the same for all values of $a$. The values of $a$ stretches and contracts the wavelet function along the time axis. The base wavelet is generated when $b=0$, and $a=1$, then the wavelet is in its natural form, which is termed the mother wavelet. The wavelet coefficients $W(a, b)$ shows the similarity between the waveform and the wavelet at a given combination of scale ' $a$ ' and position ' $b$ '.

The envelope detection or amplitude demodulation is the technique of extracting the modulating signal from an amplitude-modulated signal. The result is the time-history of the modulating signal. Envelope analysis is the FFT frequency spectrum of the modulating signal. The vibration signal of a faulty gear can be viewed as a carrier signal at a resonant frequency of the gear modulated by a decaying envelope. The goal of the enveloping approach is to replace the oscillation caused by each impact with a single pulse over the entire period of the impact.

Laplace wavelet represented by equation 4 is a complex analytical and single sided damped exponential and the view of Laplace wavelet is shown in Figure 2

$$
\psi(t)=\mathrm{A} \mathrm{e}^{-\left(\frac{\beta}{\sqrt{1-\beta^{2}}}+i\right) \omega_{c} t} \mathrm{t} \geq 0
$$

$$
\psi(t)=0 \quad \mathrm{t} \text { is otherwise }
$$

Where $\beta$ is the damping factor that controls the decay rate of the exponential envelope in the time domain and hence regulates the resolution of the wavelet and it simultaneously corresponds to the frequency bandwidth of the wavelet in the frequency domain. The frequency $\omega_{c}$ determines the number of significant oscillations of the wavelet in the time domain and corresponds to wavelet center frequency in the frequency domain and $\mathrm{A}$ is an arbitrary scaling factor [18].

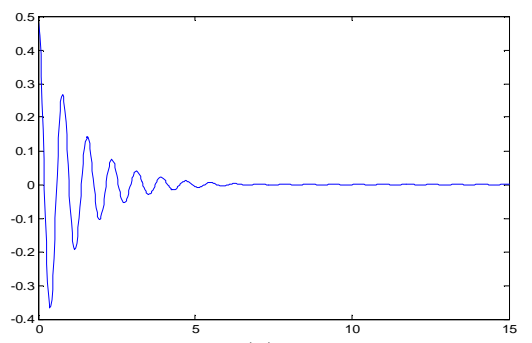

(a)

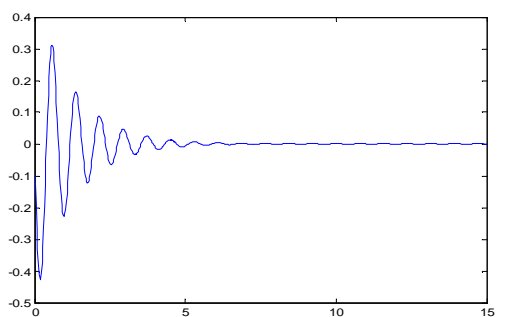

(b)

Figure 2.Laplace wavelet (a) Real part of Laplace wavelet (b) Imaginary part of Laplace wavelet

The optimal values of $\beta$ and $\omega_{\mathrm{c}}$ for given vibration signal can be found by adjusting the time-frequency resolution of the Laplace wavelet to the decay rate and the frequency of the impulses to be extracted. A high kurtosis values indicates high impulsive content of the signal with more sharpness in the signal intensity distribution

Let $x(n)$ be a real discrete time random process, and $W T_{a}$ its $N$ point Laplace wavelet transform at scale $a$. The Laplace Wavelet Kurtosis (LWK) for $x(n)$ is defined as the kurtosis of the magnitude of $W T_{a}$ at each wavelet scale $a$ as in the equation (5)[19].

$\operatorname{LWK}_{(\mathrm{a})}=\frac{\sum_{n=1}^{N} a b s\left(W T_{a}{ }^{4}\left(x(n), \psi_{\beta}, \omega_{c}\right)\right)}{\left[\sum_{n=1}^{N} a b s\left(W T_{a}{ }^{2}\left(x(n), \psi_{\beta}, \omega_{c}\right)\right)\right]^{2}}$

The objective of the Laplace wavelet shape optimization process is to find wavelet shape parameters which maximize the Laplace wavelet kurtosis.

Genetic algorithm is used to optimize the wavelet parameters. A genetic algorithm (GA) is computerized search and optimization algorithms based on the mechanics of natural genetics and natural selection. The principal idea of the GA is to search for the optimal solution in a large population. The basic steps involved in the genetic algorithm are shown below. 1. [Start] Generate random populationof chromosomes

2. [Fitness] Evaluate the fitness function of each chromosomein the population

3. [New Population] Create a new population using selection, crossover and mutation until the new population is complete

4. [Replace] Use new generated population for a further run of the algorithm

5. [Test] If the end condition is satisfied, stop, and return the best solution in current population

6. [Loop] Go to step 2

In this research roulette wheel, Arith-crossover and uniform mutation is used to find optimum solution. 
The scale-kurtosis distribution of the wavelet transform using Laplace wavelet is depicted in the Figure 3.

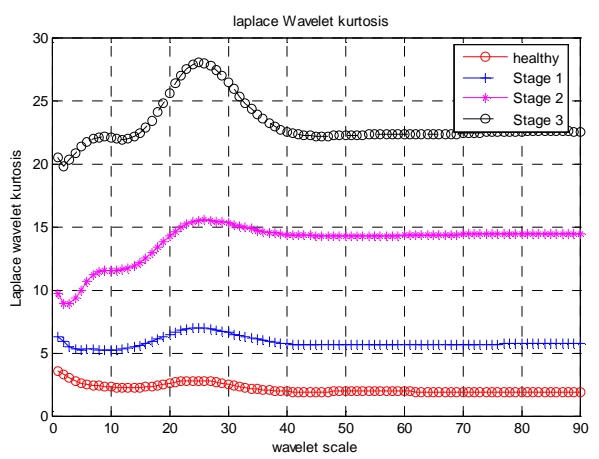

Figure 3: Scale-Kurtosis distribution

The Morlet wavelet is defined as given in equation (6)[25]. The view of Morlet wavelet is shown in Figure 4

$\psi(t)=e^{-t^{2}} \cos \left(\pi \sqrt{\frac{2}{\ln 2}} \mathrm{t}\right)$

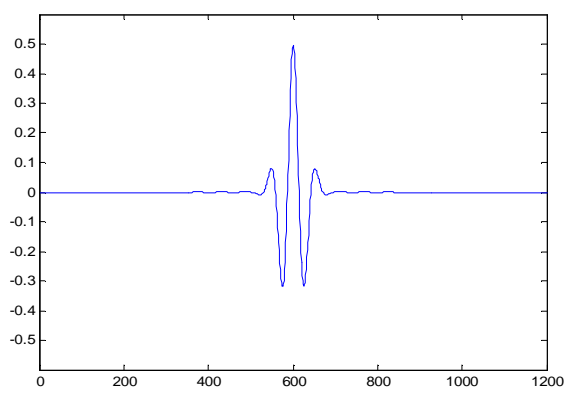

Figure4. Morlet wavelet

The wavelet transform (WT) of a finite energy signal $\mathrm{x}(\mathrm{t})$, with the mother wavelet $\psi(t)$, is the inner product of $x(t)$ with a scaled and conjugate wavelet $\psi^{*} a, b$. Since the analytical and complex wavelet is employed to calculate the wavelet transform, the result of the wavelet transform is also an analytical signal as shown in equation (7) and (8).

$$
\begin{aligned}
& \mathrm{WT}\{\mathrm{x}(\mathrm{t}), a b\}=\left\langle\mathrm{x}(\mathrm{t}), \psi_{\mathrm{a}, \mathrm{b}}(\mathrm{t})\right\rangle=\frac{1}{\sqrt{a}} \int_{-\infty}^{\infty} x(t) \psi_{\mathrm{a}, \mathrm{b}}^{*}(t) d t(7) \\
& =\operatorname{Re}[\mathrm{WT}(\mathrm{a}, \mathrm{b})]+\mathrm{i} \operatorname{Im}[\mathrm{WT}(\mathrm{a}, \mathrm{b})]=\mathrm{A}(a, b) \mathrm{e}^{i \theta(a . b)}
\end{aligned}
$$

Where $\psi_{a, b}$ is a family of daughter wavelet, defined by the dilation parameter $a$ and the translation parameter $b$, the factor $1 / \sqrt{a}$ is used to ensure energy preservation. The time-varying function $\mathrm{A}(a, b)$ is the instantaneous envelope of the resulting wavelet transform (EWT) which extracts the slow time variation of the signal, and is given by equation (9)

$$
\mathrm{A}(a, b)=\operatorname{EWT}(\mathrm{a}, \mathrm{b})=\sqrt{\{\operatorname{Re}[\mathrm{WT}(\mathrm{a}, \mathrm{b})]\}^{2}+\{\operatorname{Im}[\mathrm{WT}(\mathrm{a}, \mathrm{b})]\}^{2}}(9)
$$

For each wavelet, the inner product results in a series of coefficients which indicate how close the signal is to that particular wavelet. To extract the frequency content of the enveloped correlation coefficients, the wavelet-scale power spectrum (SWPS) (energy/unit scale) is given by equation (10)

$$
\text { SWPS }(\mathrm{a}, \omega)=\int_{-\infty}^{\infty}|S E W T(a, \omega)|^{2} d \omega
$$

Where SEWT $(a, \omega)$ is the Fourier transform of EWT $(a, b)$.

The total energy of the signal $\mathrm{x}(\mathrm{t})$ is given in equation (11)

$$
\operatorname{TWPS}=\int|x(t)|^{2} \mathrm{dt}=\frac{1}{2 \pi} \int_{-\infty}^{\infty} \operatorname{SWPS}(a, \omega) d a
$$

\section{EXPERIMENTAL SETUPS AND PROCEDURE OF DATA COLLECTION}

\subsection{Experimental Setup1}

The fault simulator setup 1consists of variable speed motor, single stage gear box, couplings, belt \& pulleys, loading system, and bearings as depicted in Figure 5. The electric motor of capacity $0.5 \mathrm{HP}$ and variable speed maximum up to $10,000 \mathrm{RPM}$ is connected to one gear of the gear box through coupling and belt \& pulleys system and the other gear is connected to a loading system. The gear and pinion has 30 and 20 teeth respectively. The steel shafts of $5 / 8$ " diameter connect gears with motor and loading system. The shafts are supported through bearings. The vibration data is collected from the drive end bearing of gear box using the accelerometer with a NI Data Acquisition Device. The views of healthy and faulty gears are shown in Figure 6. The collected vibration data are exported as data file to MATLAB software for further processing of the signal to identify the fault.

In the experimental investigation, the vibration signal was collected from a healthy gear at shaft speed of 900RPM under constant load condition. The Gear mesh frequency (GMF) is calculated to be $20 * 900 / 60=300 \mathrm{~Hz}$. Further faults were induced in two different stages as shown in Table 1 and the corresponding vibration readings were taken. The sampling frequency of $16,000 \mathrm{~Hz}$ was used to collect the data for 2 seconds. The data was collected from the setup after reaching the required speed. 


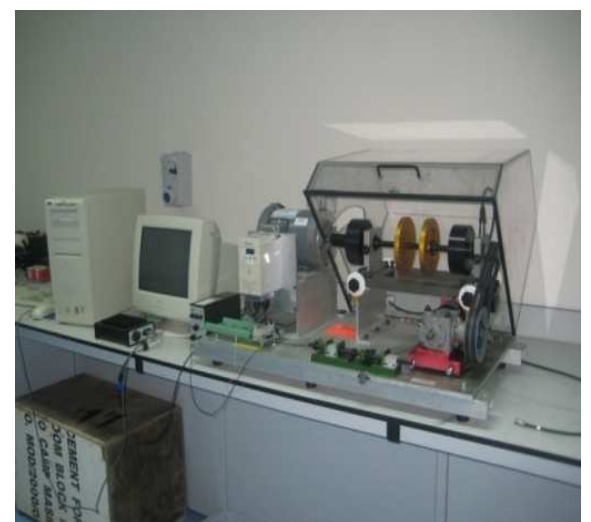

Figure5.Fault Simulator set up 1

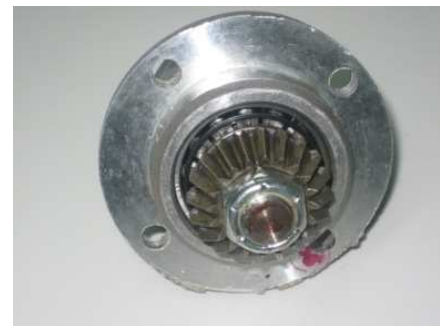

(a)

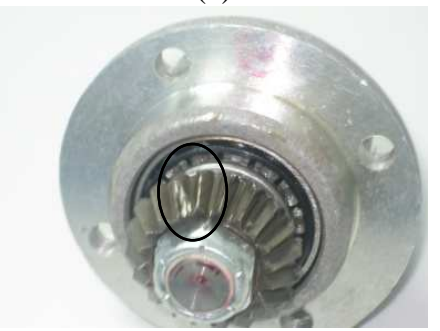

(b)

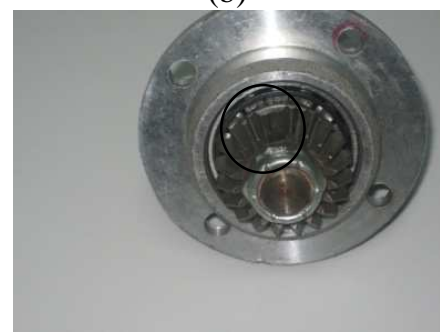

(c)

Figure 6.Gears with induced fault in 2 stages. (a) Stage 0. (b) Stage 1. (c) Stage 2.

Table 1.Stages of induced fault for Fault Simulator set up 1

\begin{tabular}{|l|l|l|}
\hline Stage & Condition of the gear & Fault description \\
\hline Stage 0 & Healthy gear & Without any induced fault \\
\hline Stage 1 & Faulty gear & The chipped tooth \\
\hline Stage 2 & Faulty gear & $\begin{array}{l}\text { Tooth was completely } \\
\text { removed }\end{array}$ \\
\hline
\end{tabular}

\subsection{Experimental Setup2}

The fault simulator2 used for the experimentation to collect the data is shown in Figure 7. It consists of a motor, single stage gear box and loading system. The gearbox input side was connected to $0.5 \mathrm{HP}, 2900 \mathrm{RPM}$ electric motor through coupling and the output side was connected to a loading system. The gear and pinion has 46 and 23 teeth respectively. The shafts of $25 \mathrm{~mm}$ diameter connect gears with motor and loading system. All drive shafts are supported at its ends with antifriction bearings. The vibration data was collected from the drive end bearing of gear box using the accelerometer (model 621B40, IMI sensors, sensitivity is $1.02 \mathrm{mV} / \mathrm{m} / \mathrm{s} 2$ and frequency range up to $18 \mathrm{kHz}$ ) with a NI Data Acquisition Device. The view of the healthy gears is depicted in Figure 8 . The vibration data collected are exported as data file to MATLAB software for further processing of the signal to identify the fault.

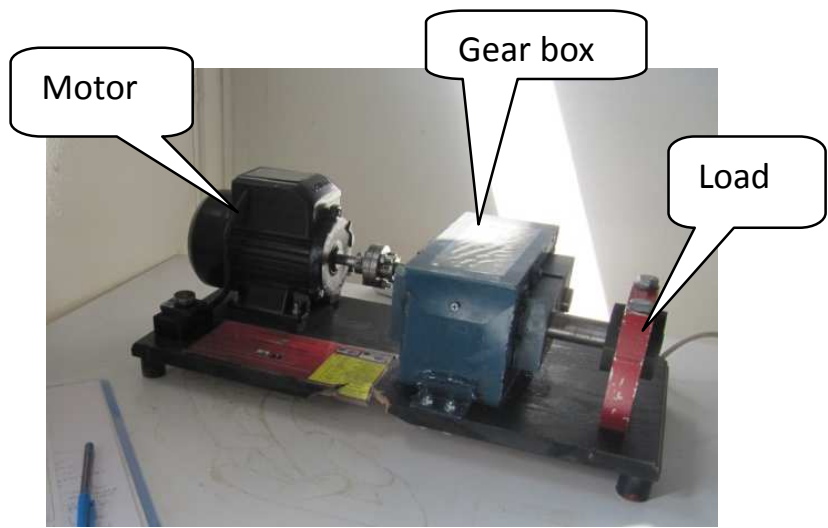

Figure 7.Fault Simulator set up 2.

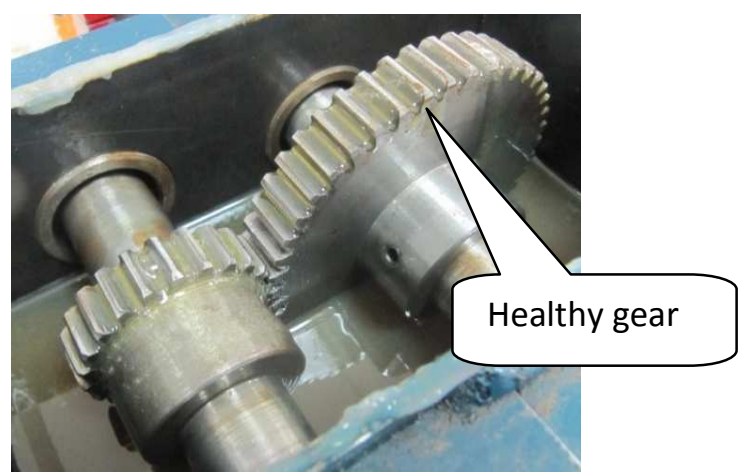

Figure 8.View of healthy gears

In the experimental investigation, the vibration signal was collected from a healthy gear at shaft speed of 2850 RPM under constant load condition. The Gear mesh frequency (GMF) is $23 * 2850 / 60=1092.5 \mathrm{~Hz}$. Further faults are induced in three different stages as shown in Table 2 and the corresponding vibration readings were taken. The views of faulty gear are shown in Figure 9. The sampling frequency of 
$16,000 \mathrm{~Hz}$ was used to collect the data for 2.4 seconds. The data was collected from the setup after reaching the required speed.

\begin{tabular}{|l|l|l|}
\hline Stage & $\begin{array}{l}\text { Condition of } \\
\text { the gear }\end{array}$ & Fault description \\
\hline Stage 0 & Healthy gear & Without any induced fault \\
\hline Stage 1 & Faulty gear & $\begin{array}{l}\text { A crack of 3mm is induced at } \\
\text { the root of the tooth }\end{array}$ \\
\hline Stage 2 & Faulty gear & Tooth was partially broken \\
\hline Stage 3 & Faulty gear & Tooth was completely removed \\
\hline
\end{tabular}

Table 2: Stages of induced fault for Fault Simulator set up 2.

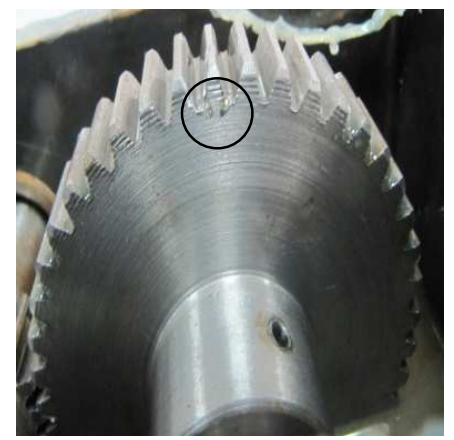

(a)

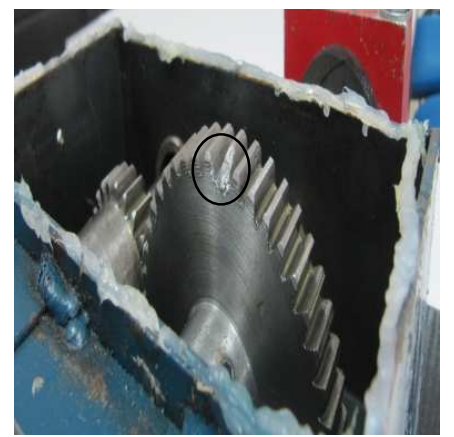

(b)

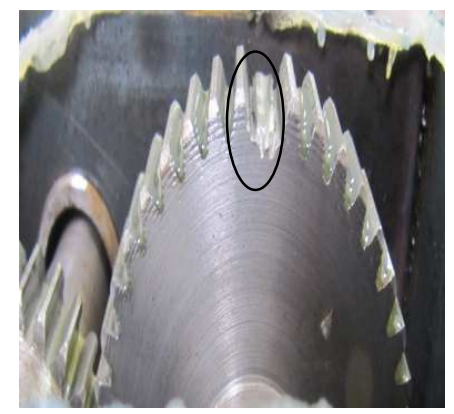

(c)

Figure9 Gears with induced fault in 3 stages. (a) Stage 1. (b) Stage 2. (c) Stage 3

\section{IMPLEMENTING OF WAVELET POWER SPECTRUM}

This section presents several application examples to visualize the performance of the proposed approach. The proposed approach is applied for the detection of localized gear fault. It is well known that the most important components in gear vibration spectra are the tooth meshing frequency and its harmonics, together with sidebands due to modulation phenomena. The increment in the number and amplitude of sidebands indicate a fault condition.

A typical time domain signal obtained from the experimental setup with gear fault, using accelerometer is given in Figure 10.The time data obtained from the setups are processed with the Wiener filter technique to enhance the signal components. This is further processed using various signal processing techniques like wavelet enveloped power spectrum based on Morlet wavelet and Laplace wavelet. The GMF and its side bands are represented in various power spectrums with indication of data cursor value.The rotational frequency of pinion, gear, GMF and some of theside bands of GMF are depicted in Table 3 for both the experimental setups.

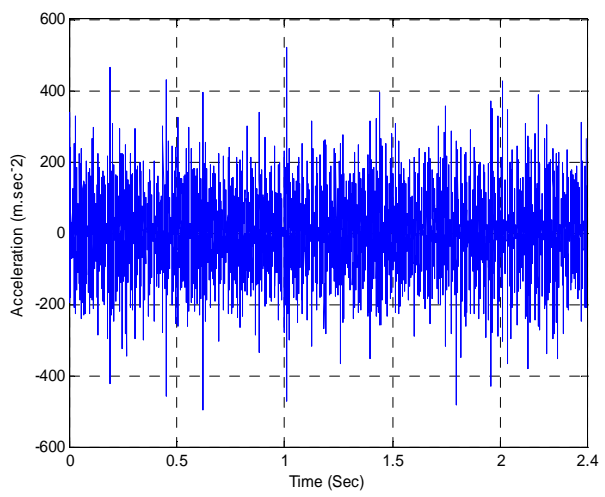

Figure 10.Time domain signal of gear with fault

Table 3.Frequency of rotation

\begin{tabular}{|l|l|l|}
\hline Description & $\begin{array}{l}\text { Experimental set } \\
\text { up 1(Frequency) }\end{array}$ & $\begin{array}{l}\text { Experimental set } \\
\text { up 2(Frequency) }\end{array}$ \\
\hline $\begin{array}{l}\text { Pinion rotational } \\
\text { frequency }\end{array}$ & 48 & 15 \\
\hline $\begin{array}{l}\text { Gear rotational } \\
\text { frequency }\end{array}$ & 24 & 10 \\
\hline GMF band & 1093 & 300 \\
\hline $\begin{array}{l}\text { Side band } \\
\text { frequency at GMF }\end{array}$ & 1140 & 285 \\
\hline $\begin{array}{l}\text { Side } \\
\text { frequency at GMF }\end{array}$ & 290 \\
\hline
\end{tabular}




\subsection{Laplace Wavelet Enveloped Power Spectrum for the Experimental Set Up 1}

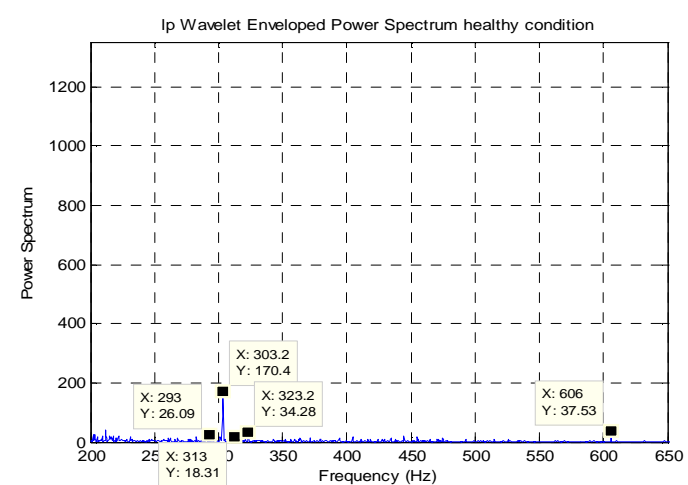

(a)

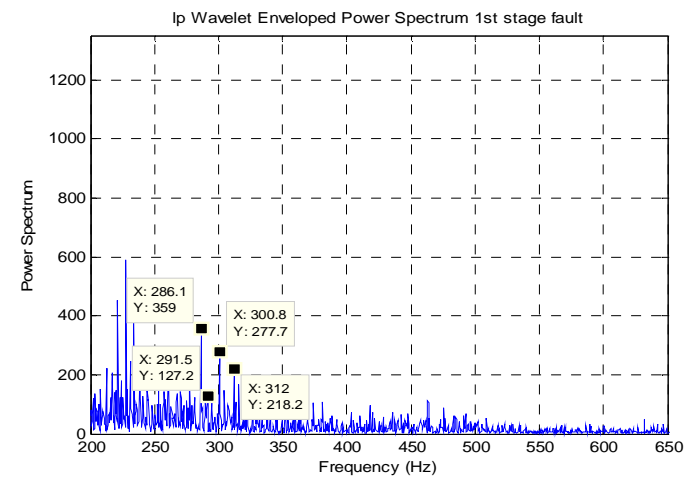

(b)

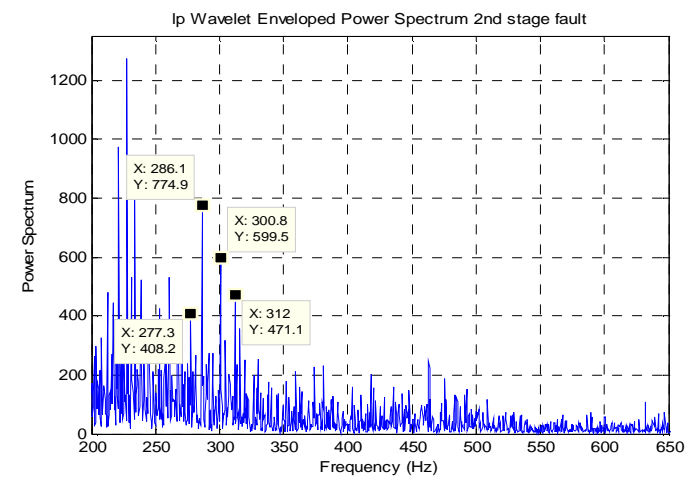

(c)

Figure 11.Laplace wavelet enveloped Power Spectrums for experimental set up 1. (a) Without any defect. (b) $1^{\text {st }}$ stage of defect. (c) $2^{\text {nd }}$ stage of defect

Figure 11 (a) to 11(d) depicts that vibration amplitude at GMFis increasing in line with the severity of fault from 170 to $599 \mathrm{~mm} / \mathrm{s}^{2}$ with dominant sidebands. Side bands in the spectrum indicate the severity of the fault. The side bands level remains low for healthy condition of gears and the side bands are risingwith the severity of the fault.

\subsection{Laplace Wavelet Enveloped Power Spectrum for the Experimental Set Up 2}

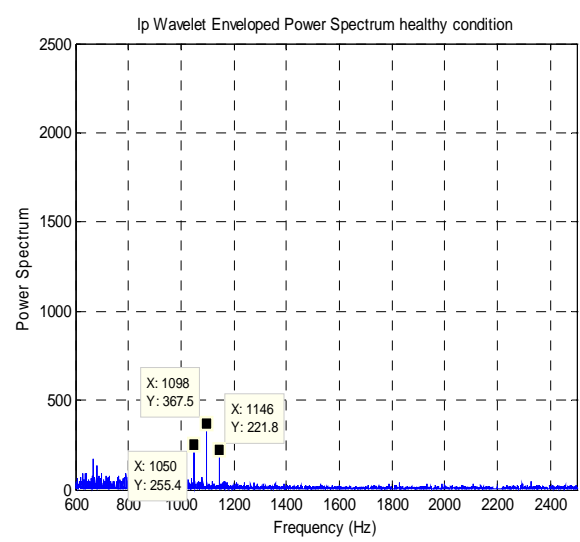

(a)

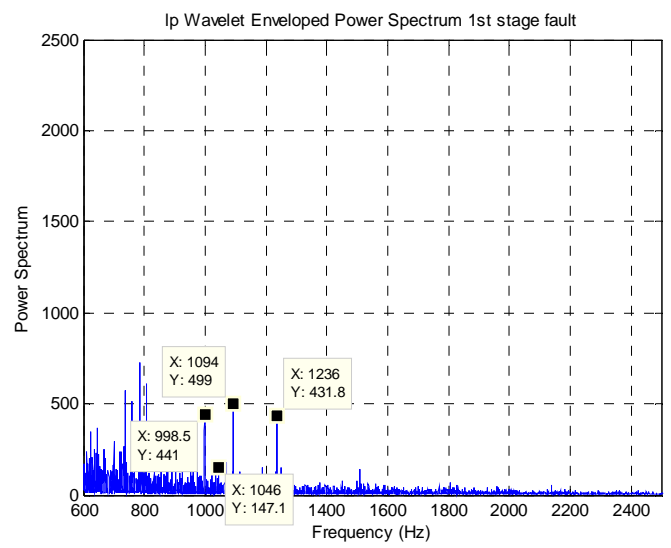

(b)

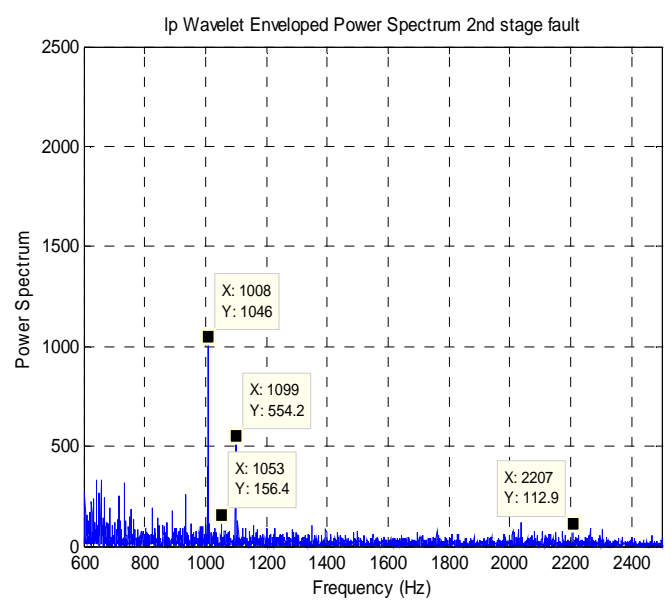

(c) 


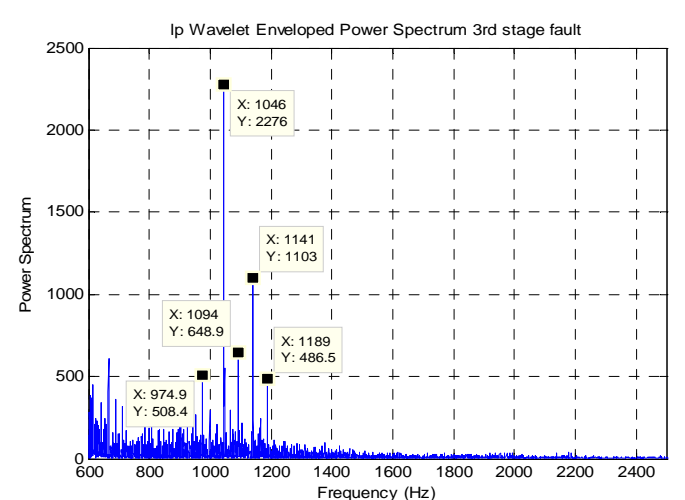

(d)

Figure 12.Laplace wavelet enveloped Power Spectrums for experimental set up 2. (a) Without any defect. (b) $1^{\text {st }}$ stage of defect. (c) $2^{\text {nd }}$ stage of defect(d) $3^{\text {rd }}$ stage of defect

Figures 12(a)-12(d) depicts the Laplace wavelet enveloped power spectrums for the healthy and faulty gear in three different stages of fault for the experimental setup2. The vibration amplitude at GMFis increasing in line with the severity of fault from 367.5 to $648 \mathrm{~mm} / \mathrm{s}^{2}$ with dominant sidebands, indicating severity of fault. Side band level remains low as seen in Figure 12(a), for a gear box in good condition.

\subsection{Morlet Wavelet Enveloped Power Spectrum for}

\section{Experimental Set Up 1:}

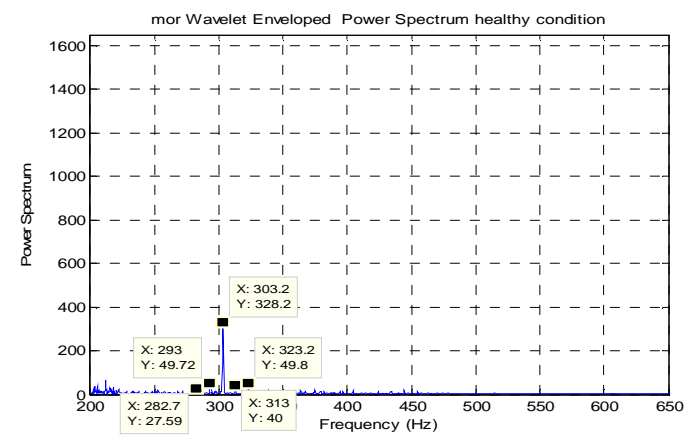

(a)

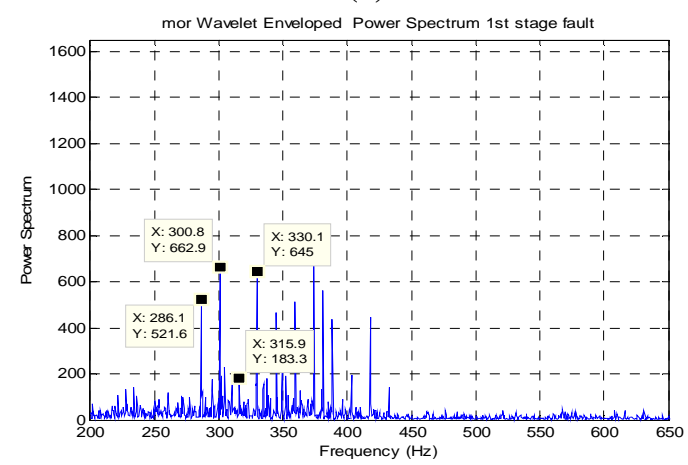

(b)

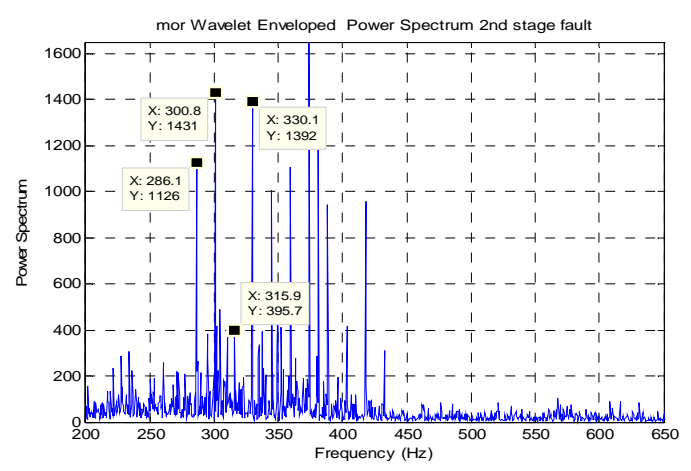

(c)

Figure 13.Morlet wavelet enveloped Power Spectrums for experimental set up 1. (a) Without any defect. (b) $1^{\text {st }}$ stage of defect. (c) $2^{\text {nd }}$ stage of defect

The vibration data of the experimental set up 1used to develop the Morlet wavelet enveloped power spectrums are shown in Figure 13(a) - 13(d). Amplitude of GMF is increasing from 328 to $1431 \mathrm{~mm} / \mathrm{s}^{2}$ for healthy gear and faulty gear in 2 different stages. The significant increase in amplitude of side bands above the GMF amplitude indicates the severity of fault.

\subsection{Morlet wavelet enveloped Power Spectrum for the}

\section{experimental set up 2}

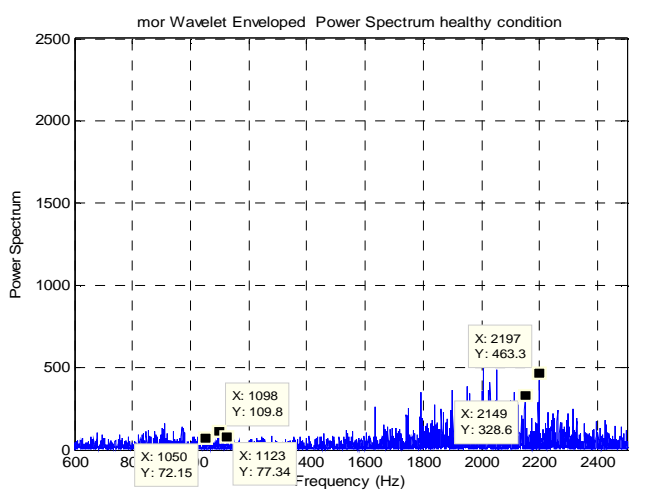

(a)

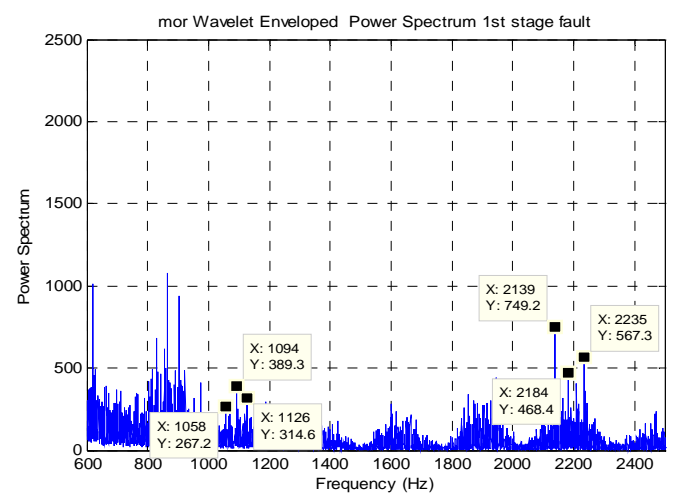

(b) 


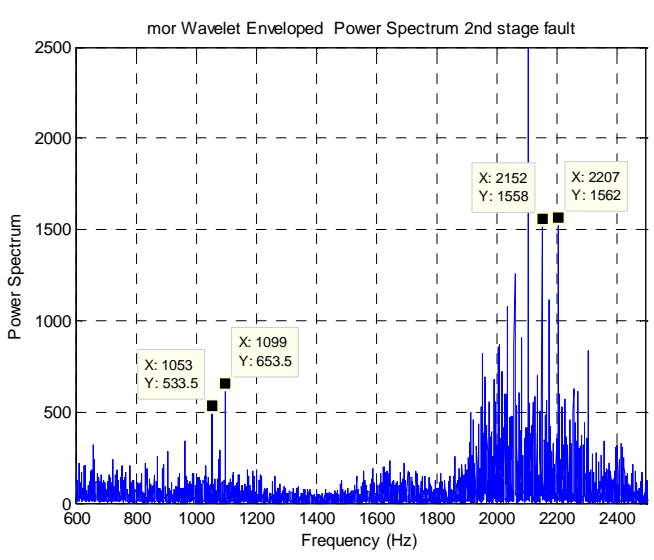

(c)

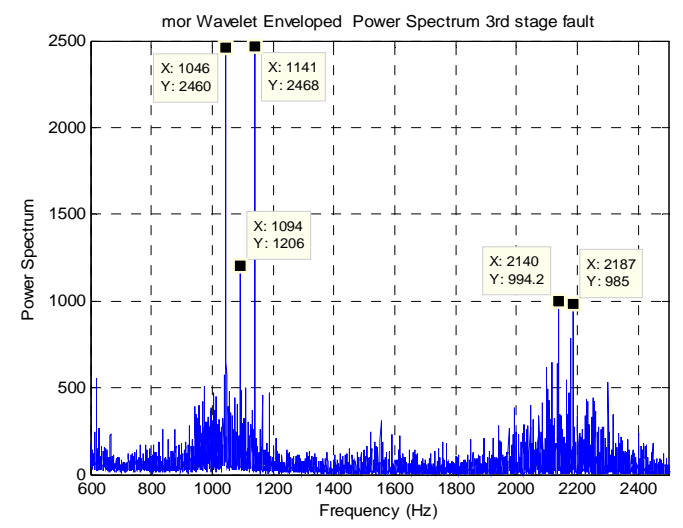

(d)

Figure 14.Morlet wavelet enveloped Power Spectrums for experimental set up 2. (a) Without any defect. (b) $1^{\text {st }}$ stage of defect. (c) $2^{\text {nd }}$ stage of defect (d) $3^{\text {rd }}$ stage defect

Morlet wavelet enveloped power spectrums developed for the experimental set up2 data are shown in Figure 14(a)-14(d). Amplitude at GMF is increasing from 109 to $1206 \mathrm{~mm} / \mathrm{s}^{2}$ for healthy gear and faulty gear in 3 different stages.Vibration amplitude of side bands are significant. Changes in the number and strength of the side bands are noticed.

\section{LAPLACE WAVELET TRANSFORM AND ANN FOR FAULT CLASSIFICATION}

Although analysis of wavelet enveloped power spectrum by an experienced technical person is possible, computerized inspection is easier and recommended for the increasing demand for on-line automated condition monitoring applications. Artificial neural network (ANN) is used for an automated detection and diagnosis of gear conditions. To speed up the classification process and to make it suitable for the online condition monitoring, the data processed using Laplace-wavelet transform is used as input to ANN. The extracted features in time and frequency domain are used as the ANN input vectors for the gear condition identification.
The ANN classifier parameters are optimized using GA by minimizing the mean square error (MSE).

As shown in Figure 3, the scale-kurtosis distribution for different gear conditions, it could be seen that the scales range of 20-30 are the mostly dominant scales, which can reveal the gear condition sufficiently. The predominant Laplace wavelet transform scale based on the scale-kurtosis value has been selected for features extraction.

The four extracted features in time and frequency domain for the dominant scale are:

1) Time domain features: This includes Standard Deviation (SD), and Kurtosis factor(KF).

2)Frequency domain features: This includes the ratio of SWPSpeak frequency $\left(f_{\max }\right)$ to the shaft rotational frequency $\left(f_{r p m}\right)$, and the ratio of SWPS maximum amplitude $\left(A_{\max }\right)$ to the overall amplitude (Sum $\left(A_{i}\right)$ ).

The scheme of feature extraction and overall architecture of ANN is depicted in Figure 15.

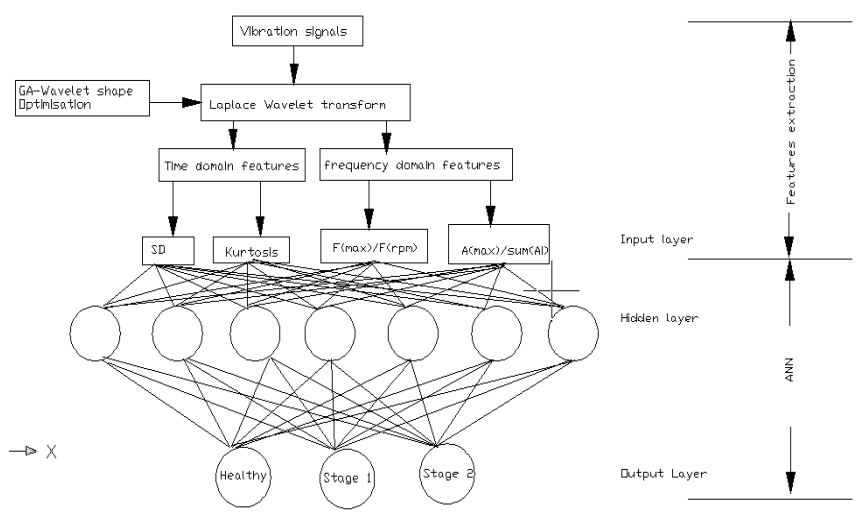

Figure 15: features extraction and ANN architecture

A feed-forward multi-layer perceptron (MLP) neural network with three layers has been developed namely Input layer, Hidden layer and output layer. The features extracted from the predominant scale of Laplace wavelet transform in time and frequency domain are used as input to ANN with four source nodes. The hidden layer with seven computation nodes has been used. The number of the hidden nodes is optimized using a genetic algorithm by minimization of Mean Square Error (MSE) between the actual network outputs and the corresponding target values. The output layer has three nodes to represent working conditions of gear.

The three digit output target nodes need to be mapped by the ANN are represented as $[1,0,0]$ for the healthy condition, $[0,1,0]$ for the stage 1 , and $[0,0,1]$ for stage 2 of gear. 
The activation function $f_{1}$, bias $b$ and weight $w$ are used to transform the input vector to intermediate vector $h$ and the output vector $O$ of the network is obtained from the vector of the intermediate variable $h$ through a similar transformation using activation function $f_{2}$ at the output layer. The connection weights and biases are modified iteratively to optimize the performance criterion, while training the network. Commonly used minimization of mean square error(MSE) is used as the performance criterion. The process is repeated until the overall MSE value drops below some pre-determined threshold (stopping criterion). After the training process, the ANN weights are fixed and the system is deployed for the unseen vibration data.

In this work MATLAB software is used for ANN process with Levenberg-Marquarat Back-propagation (LMBP) training algorithm, a MSE of 10E-10, a minimum gradient of 10E-20 and maximum iteration (epochs) of 1000 were used. The training process would stop if any of these conditions were met. The initial weights and biases of the network were generated automatically by the program

\subsection{Implementing WT and ANN}

The data collected from the experimental setup with different working conditions are used to train and test the ANN. The neural network input feature vectors consisting of four groups representing the different gear conditions are used to train the network. The data sets of size 1920 samples each is used to train the network. The parameters used for the network are shown in Table 4

Table 4: Parameters used for the network

\begin{tabular}{|l|l|}
\hline Transfer Function of Hidden Layer & Sigmoid \\
\hline Transfer Function of output Layer & Linear \\
\hline Training Algorithms & $\begin{array}{l}\text { Levenberg- } \\
\text { Marquarat }\end{array}$ \\
\hline Maximum number of epochs & 1000 \\
\hline MSE stopping criteria & $1 \mathrm{e}-10$ \\
\hline Minimum performance gradient & $1 \mathrm{e}-20$ \\
\hline Number of input nodes & 4 \\
\hline Number of hidden nodes & 7 \\
\hline
\end{tabular}

The training can ceaseaccording to any one of the criteria of mean square error (MSE), the number of epochs of training or minimum performance gradient reaches certain value set as shown in Table 4. The result of ANN learning processhas R value of above 0.99 for training, testing and validation, which is the indication of the relationship between the outputs and targets of ANN The ANN learning process and the performance plot is depicted in Figure 16, which shows that the training with 82 epochs met the MSE stopping criteria (MSE less than 10E-10).
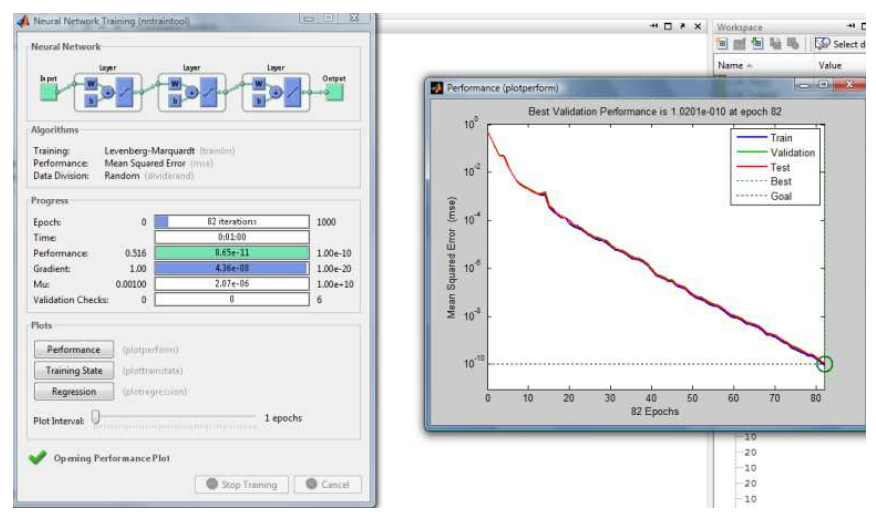

Figure 16: ANN Learning process and performance plot

The ideal output target nodes needs to be mapped by the ANN are represented as $[1,0,0]$ for the healthy condition, $[0,1,0]$ for the stage 1 , and $[0,0,1]$ for stage 2 of gear condition is depicted in Figure 17(a)and the testing process is depicted in Figure (b).The successful classification of ANN test process for unseen vibration data of the trained ANN for the healthy, stage 1 and stage 2 of gear condition are shown in Figure17(c)17(e) respectively.

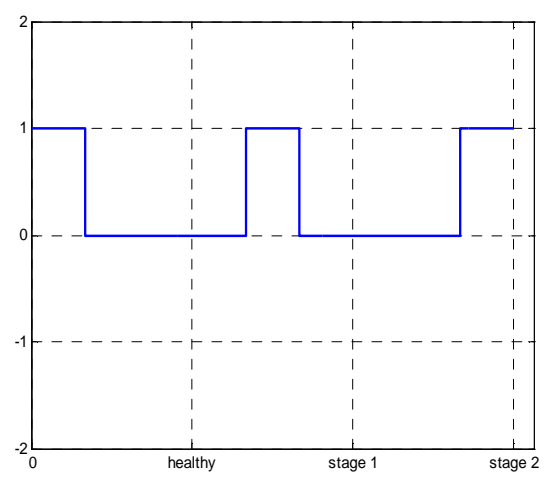

(a)

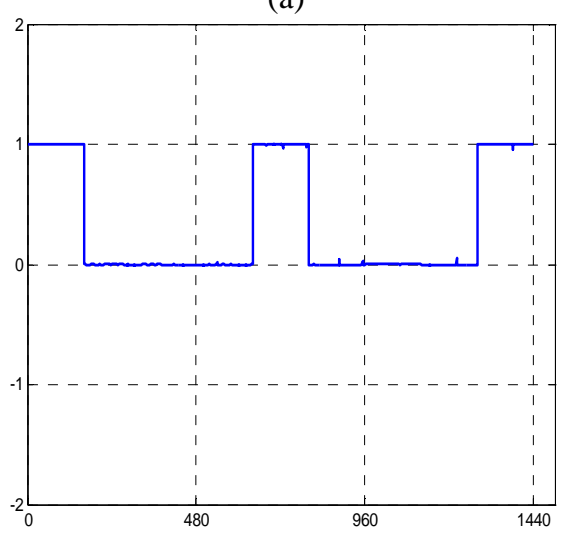

(b) 


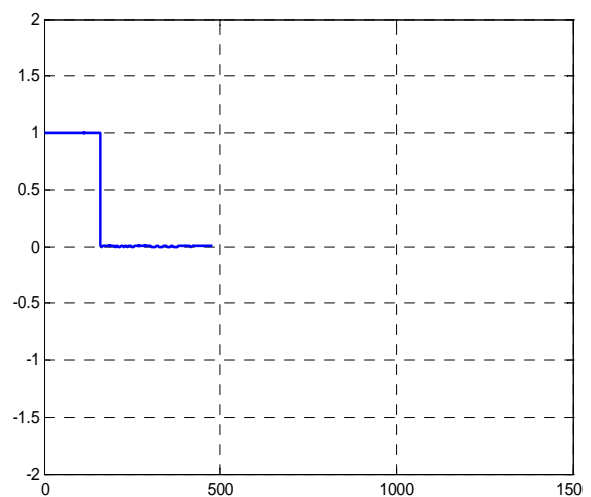

(c)

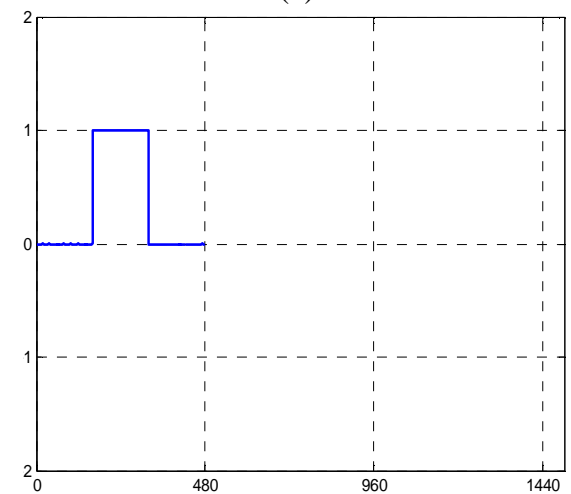

(d)

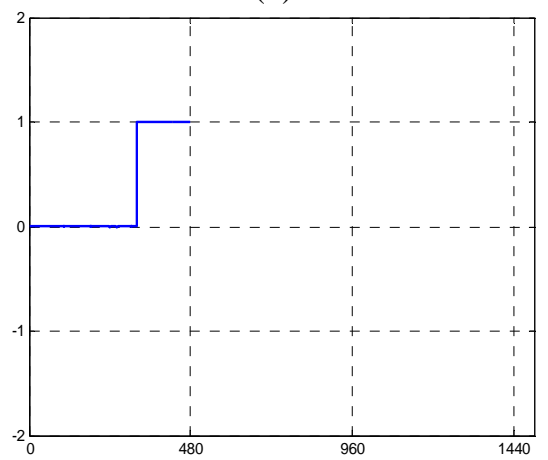

(e)

Figure 17: ANN train and testing process (a) Training process

(b) Testing process (c) Test process for unseen healthy data

(d) Test process for unseen stage1 data(e) Test process for unseen stage 2 data

\section{CONCLUSIONS}

Wavelet enveloped power spectrum and ANN used for gear fault detection and diagnosis is presented in this paper.Various stages of induced fault and healthy conditions of gear are studied using Laplace and Morlet wavelet based envelop power spectrum. Wiener filter is used toenhance the fault signal components, which support for the early detection of the fault.
Further, the studies conducted indicates that both Morlet and Laplace wavelet based enveloped power spectrum depicts significant increase in magnitude of vibration amplitude at GMF along with increase inthe size of fault.Laplace wavelet analysis is more powerful inisolating peaks at multiple RPM sidebands of GMF which canprovide more precise information about defect condition. Wavelet and ANN parameters are optimized using Genetic algorithm. Features extracted from optimized wavelet scale are used as input vectors to ANN.The application of proposed technique shows its effective detection and classification of ANN test.

\section{REFERENCES:}

[1] WandelJ.. 2006. Multistage gearboxes: Vibration based quality control, KTH Engineering Sciences, Stockholm, Licentiate Thesis, TRITA-AVE 2006:27 ISSN1651-7660.

[2] M.E.M. El Badaoui, F. Guillet, J. Daniere, New applications of the real cepstrum to gear signals: including definition of a robust fault indicator, Mechanical Systems and Signal Processing 18 (5) (2004) 1031-1046.

[3] W. Staszewski, K. Worden, G.R. Tomlinson, Timefrequency analysis in gearbox fault detection using the Wigner-Ville distribution and pattern recognition, Mechanical Systems and Signal Processing 11 (5) (1997) 673-692.

[4] Abu-Mahfouz I.A., 2007. Gearbox health monitoring experiment using vibration signals. Journal of Engineering Technology, Vol.24, No. 1, pp. 26-31.

[5] Omar F.K. and Gaouda A.M.,2009. Gearbox diagnostics using wavelet based windowing technique. Journal of Physics: Conference Series, Vol. 181, No.No. 1, Conf. Ser. 181012089 [6] Lin J, Zuo M.J., 2003. Gear box fault diagnosis using adaptive wavelet filter,Mechanical Systems And Signal Processing, Vol. 17,No. 6, pp. 1259-1269.

[7] Saravanan N. Ramachandran K.I.,.2010. Incipient gear box fault diagnosis using discrete wavelet transform for feature extraction and classification using artificial neural network An international journal of Expert Systems with Applications, Vol. 37, pp.4168-4181

[8] Choy F.K., Huang, J.J., Zakrajsek R.F, Handschuh T.P, Townsend.1994.Vibration signature of a faulted gear transmission system NASA, Technical Memorandum 106623, Technical Report ARL-TR-475 AIAA-94-2937

[9] Dalpiaz G., Rivola A. And Rubini R.,1998 Gear fault monitoring: comparison of vibration analysis techniques Proceedings of the 3rd International Conference on Acoustical and Vibratory Surveillance Methods and Diagnostic Techniques, Vol. 13, pp, 623-637

[10] Randall R.B. 1982. A new method of modeling gear faults. Journal ofMechanical Design, Vol. 104, pp. 259-267

[11] Pan M.C., Sas P., 1996. Tansient analysis on machinery condition monitoring International Conference on Signal Processing, Proceedings, ICSP 2,pp. 1723-1726.

[12]Peng Z.K., Chu F.L., 2004 Application of the wavelet transform in machine condition monitoring and fault 
diagnostics: a review with bibliography. Mechanical Systems and Signal Processing, Vol. 18, No. 2, pp. 199-221

[13] Purushotham V., Narayanan S, and Suryanarayana, Prasad A.N., 2005Multi-fault diagnosis of rolling bearing elements using wavelet analysis andhidden Markov model based fault recognition. NDT \& E International Vol. 38,No. 8, pp. 654-664.

[14] Shi D.F., Wang W.J. and Qu L.S., 2004. Defect detection for bearing using envelope spectra of wavelet transform. ASME J of Vibration and Acoustics, Vol.120, pp. 567-574.

[15] Freudinger L.C., Lind R., and Brenner M.J., 1998. Correlation filtering of modal dynamics using the Laplace wavelet Proceeding of the 16th International Modal Analysis Conferences, Vol. 2, pp. 868-877.

[16] Yanyang Z., Xuefeng C. Zhengila H. And Peng C., 2005. Vibration basedmodal parameters identification and wear fault diagnostics using Laplacewavelet. Key Engineering Materials, Vol. 293-294, pp. 183-190.

[17] Juan Jos'éGonz'alez de la Rosa, Member, IEEE, A. Moreno, A. Gallego, R. Piotrkowski and E. Castro "Termites detection via spectral kurtosis and waveletde-noising of acoustic emission signals"journal of acoustic emission, 26, 2008.

[18]Al-Raheem K.F, Roy. A, Ramachandran.K.P. 2006. Detection of rollingelement bearing faults using optimized wavelet denoising technique.

International Conference on Signal Processing, Beijing, China [19]Al-Raheem K.F., Roy A., Ramachandran K.P, Harrison D.K., Grainger S.,2009, International Journal of Advanced Manufacturing Technology, Vol. 40, pp.393-402.

[20] Al-Raheem K.F., Roy A., Ramachandran K.P, Harrison D.H., Grainger, S.2007. Rolling element gearing fault diagnosis using Laplace-wavelet envelop power spectrum EURASIP Journal on Advances in Signal Processing, Vol. 2007, articleid 73629, 14pages

[21]S.N.Sivanandan, S.Sumati, S.N.Deepa, Introduction to neural network using Matlab 6.0, Mcgraw hill education (India) private limited, 2006, ISSN:978-0-07-059112-7

[22] J. Rafieea,_, F. Arvania, A. Harifib, M.H. Sadeghic (2007)Intelligent condition monitoring of a gearbox using artificial neural network; Mechanical systems and signal processing;21:1746-1754

[23]B. Samanta, K.R. Al-Balushi, Artificial neural network based fault diagnostics of rolling element bearings using timedomain features, Mechanical Systems and Signal Processing 17 (2) (2003) 317-328.

24] W. Bartelmus, R. Zimroz, H. Batra, Gearbox vibration signal pre-processing and input values choice for neural network training,Artificial Intelligence Methods, Gliwice, Poland, 5-7 November, 2003.

[25] Semmlow J.L. 2004.Bio signal and biomedical image processing Marcel Dekker, Inc.New York, USA, ISBN08247-4803-4.

\section{BIOGRAPHIES:}

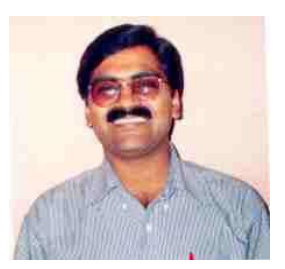

Mr. M. Lokesha received his B.E. degree in Mechanical Engineering from Mysore University, Mysore, India in1989 and M.TECH. Degree in Maintenance engineering from Mysore University, Mysore, India in 1994.Presently, he is working as a Senior Lecturer in the Department of Mechanical and Industrial Engineering at Caledonian College of Engineering, Muscat. He has been working for 22 years in engineering institutions. His research focuses on vibration instrumentation \&measurement, condition monitoring of rotating machinery. $\mathrm{He}$ is currently $\mathrm{PhD}$ research student at National Institute of Technology, Durgapur, India.

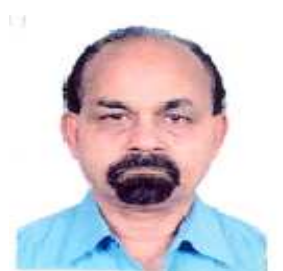

Dr. Manik Chandra Majumdar is a Professor in the Department of Mechanical Engineering and Member, Board of Governors of NIT Durgapur India. He has a $\mathrm{PhD}$ from the Indian Institute of Technology, Kharagpur, India. He has guided many Ph.D. scholars. His prime area of research is Tribology /Design \&Production

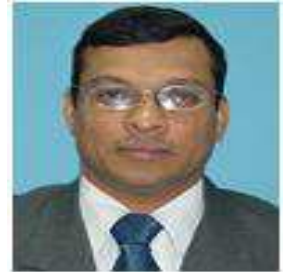

Dr. K. P. Ramachandran B.E, M.Tech, PhD is currently working as Associate Dean (Post Graduate Studies \& Research), Caledonian College of Engineering, Muscat, Sultanate of Oman. He has been working for more than 25 years in engineering institutions and as a consultant for many industries. He has research interest in the vibration instrumentation \&measurement, analysis and control, condition monitoring of rotating machinery. He has many publications to his credit and has been conferred Sir C.V. Raman award for the best technical paper published in the Journal of Vibration \& Acoustics (1997). He is on the editorial board and technical reviewer for many international journals and conferences. He has guided many $\mathrm{PhD}$ students in the area of condition monitoring and maintenance management.

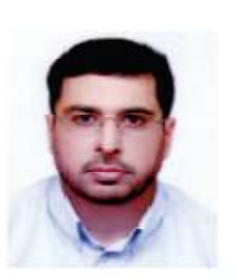

Dr. Khalid Fatihi Abdul-Raheem, B.E., M.Tech from University of Technology, Iraq and $\mathrm{PhD}$ from Glasgow Caledonian University, UK Presently he is working as a Associate professor in the Department of Mechanical Engineering at Sohar University, Sohar, Oman. He has been working for more than 22 years in engineering institutions and many industries He has many publication sto his credit. He has research interest in condition monitoring and signal analysis, 
artificial intelligence, automatic control system, mechanical vibration analysis and control. 